\title{
The Issues of Coverage in Directional Sensor Network
}

\author{
Shreya Mishra, \\ Department of Computer Science \\ Engineering \\ Dehradun Institute of Technology \\ University, Dehradun
}

\author{
Ramakant Sharma \\ Department of Computer Science \\ Engineering \\ Dehradun Institute of Technology \\ University Dehradun
}

\author{
Siddharth Saxena \\ Department of Computer Science \\ Engineering \\ Dehradun Institute of Technology \\ University, Dehradun
}

\begin{abstract}
The wireless sensor networks (WSNs) come into prominence because they have the capability of changing our economy and life. A very vast range of applications supported by WSNs has served the human society very effectively; and, monitoring has been proven the most attention drawing application among these. As a tributary of sensor networks, Directional sensor networks (DSNs) consist of several camera sensors where nodes cover only a fraction of surrounding defined by a specific angle called vertex angle at a time. As sensors are randomly deployed, their sensing direction may overlap with one another and hence coverage optimization has become the most popular area for research over the years. In this paper, the problem of coverage is surveyed on the basis of the solutions proposed, and hence, classified into three categories as (1) Target coverage (2) Maximal coverage (subset of area coverage ) and (3) Maximum coverage with minimum sensors (subset of target coverage). The impact of various parameters of directional sensor networks as: sensor's location, its working direction, number of orientations, FoVs on the performance of the deployed sensor network is analyzed. Maximum coverage with minimum sensors (subset of target coverage)
\end{abstract}

\section{Keywords}

Directional Sensor Network (DSNs), Wireless Sensor Network (WSNs), Coverage, motility.

\section{INTRODUCTION}

Research on WSNs has becomes more popular nowadays, since more powerful embedded platforms with high capabilities are being designed at rapidly decreasing costs. Moreover, multimedia capability has been added to sensor nodes with the improvement on micro electro mechanical systems (MEMS). As a variant of WSNs, DSNs are comprised of many directional sensor nodes however, unlike traditional sensors directional sensors have sector like sensing area. Any directional sensor node has a fixed angle of view and can have multiple directions but only one valid specified direction at a time. The direction in which nodes focus is termed as their working direction. The coverage region is determined by both its location and the working direction. In DSNs, the coverage is severely affected by the deployment policies. Basically, there are two deployment process i.e. deterministic deployment where the Deployment of nodes are planned and random deployment where the nodes are randomly deployed. In most practical applications random deployment of sensor nodes are used. Due to the random deployment, the sensing ranges of nodes may overlap which reduces the effective coverage area of the nodes. Thus, the coverage optimization is attracting more and more attention of the researchers. Coverage can be broadly studied in two classes i.e. area coverage and target coverage. In. area coverage the performance is measured in terms of the area covered whereas in target coverage performance is measured in terms of the number of target covered. To increase the coverage area; either the nodes mobility where the nodes have relocated to the new location can be exploited or nodes motility where re-adjustment of nodes orientation angle have been done, can be used.

Furthermore, the paper is organized as follows: In Section II, the Related Works to DSNs are discussed along with the respective experimental results and in Section III, the paper is concluded.

\section{RELATED WORK}

In practice the coverage usually involves two basic sides [1]:

- How to evaluate the coverage performance when sensor nodes are deployed in a monitoring region.

- How to improve the coverage performance when wireless sensor network cannot effectively satisfy application requirements.

Moreover, the various criteria that affect the coverage can be defined as follows:

- Deployment strategy: In DSNs, the sensor nodes can be deployed in two ways: Random deployment and Deterministic Deployment. In Random deployment, the nodes are scattered randomly and the possibility of overlapping and occlusion effect is more and in Deterministic Deployment or Planned Deployment, sensor nodes are placed at estimated locations.

- Sensing Model: Sensing model is used to estimate the capability or the ability of the deployment. It can be either binary or probabilistic. In binary model sensor can either detect the target or not detect the target. In probabilistic Model, as the distance of the target increases from the sensor its possibility of detection decreases.

- Sensing area: Sensing area refers to the area covered by sensor nodes. With the increasing sensing area greater coverage can be achieved that is the area covered by the given number of sensors [9].

- Communication Range: Communication Range is an area through which the node is able to communicate. Communication Range is invariable in most of the application. It is generally assumed as twice the sensing radius as: $\mathrm{Rc}>=2 \mathrm{Rs}$ where $\mathrm{Rc}$ is communication range and $\mathrm{Rs}$ is sensing radius.

- Sensor's Mobility: Sensor's mobility is very crucial aspect of the sensor networks as it heals many issues related to coverage and connectivity. The nodes mobility is relocation of nodes therefore, the nodes can be moved to the location where some nodes may 
run out-of battery or got damaged due to some environment disaster.

- Sensor's Motility: As sensor nodes are very scarce in resources such as power; Sensor's motility is best when exploited to minimize the overlapping and to enhance the coverage. It basically described by pan, tilt or zoom motions of the camera i.e. without relocating the node the sensors can be rotated around the axis to the new direction.

The studies and their respective solutions that have been done to enhance the coverage area of the sensor network can be classified as follows:

- $\quad$ Target coverage

- Maximal coverage.

- Maximal coverage with Minimum Sensors

\subsection{Target coverage}

Deployment can be point/target oriented or it can be location oriented. Target coverage means covering the critical points .Target coverage problem is considered as NP-Complete by many researchers. In Target coverage the generalized approach is to divide the sensors into subset or set of sensors that continuously monitor the targets. This approach is preferred due to the scarcity of the resources in sensor nodes as the major limitation of sensor nodes is its small power backup.

It can be used for the barrier coverage where the objects penetrating into region is tracked. In [2], the authors detect an algorithm based on learning automata to detect minimum number of nodes to detect the barrier.

An energy efficient heuristic for target coverage problem is proposed in [4].Authors devise an algorithm an efficient maximum coverage heuristics ( $\mathrm{MCH}$ ).It consist of a granularity parameter $\mathrm{w} \in(0,1)$ is a cover that generate contain the set of sensors say $\{\mathrm{s} 1 \ldots . . \mathrm{sn}\}$ to cover the target and then assigning a lifetime and priority to the cover and defines as the sensor network density increases with the network lifetime and more targets are covered with the more sensors.

In [4], the target coverage can be categorized into:

1) Disjoint and non disjoint

2) Target coverage with adjustable sensing region

3) Target coverage problem with QoS constraint

4) Incorporating connectivity in target coverage problem

\subsubsection{Disjoint and non- disjoint}

In disjoint approach, the sensors are categorized into disjoint subset of sensors where sensors can participate in only one set whereas in non disjoint approach, the sensors are classified into non disjoint subsets where one node can participate in more than one set at a time.
Coverage Problems in DSNs

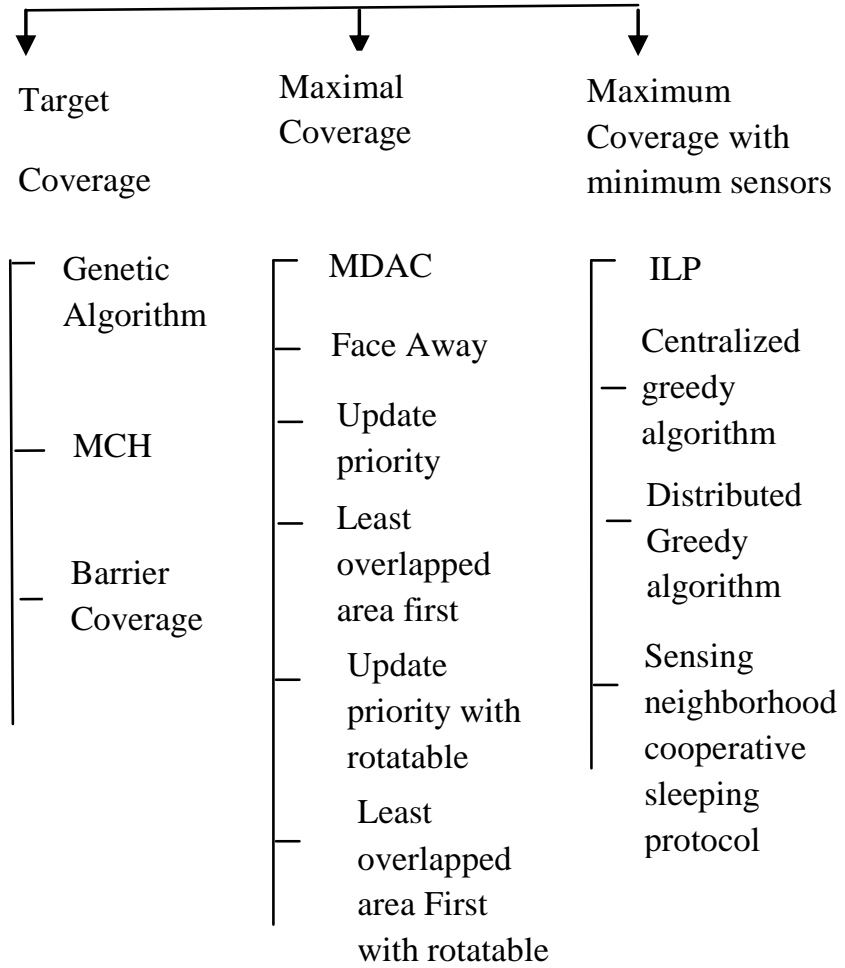

Fig 1 Classification of coverage problem in DSN

\subsubsection{Target coverage with adjustable sensing region}

Each node has multiple orientations therefore, according to the requirement, at a time one sensing direction can be chosen.

\subsubsection{Target coverage problem with QoS constraint}

QoS (Quality of service) of any network defines effectiveness of any network. In DSNs, the requirement is either to increase the coverage ratio i.e. number of target covered divided by the total number of target or by increasing coverage rate.

\subsubsection{Incorporating connectivity in target coverage problem}

The network is said to be connected when any node is able to communicate with any other node .Any node can communication with each other or with base station either periodically or continuously. The main objective is to maximize the network lifetime with minimum number of the sensors.

In [5], the number of sensors and the orientation is studied and accordingly an algorithm is proposed to provide $\mathrm{K}$-coverage. The solution given by [5] is that to cover half of the targets at most $\mathrm{M} * \log (\mathrm{K}|\mathrm{C}|)$ where $|\mathrm{C}|$ is the maximum 
number of target points covered by a sensor and $|\mathrm{M}|$ is the minimum number of sensors required to K-cover All the given points. Target may have incorporated priority; it might depend on the distance because due to the increasing distance the quality degrades. Hence for such sort of problems Jan Wan [6] proposed a genetic algorithm for approximating the minimum subset of the directional sensors required to monitor the target by considering the priorities. The algorithm defined in [6]; considers the incorporated priorities of the individual nodes and the distance between the nodes and the targets.

\subsection{Maximal Coverage}

There were various solutions devised to enhance the area coverage of the sensor networks and the impact of how the number of orientation, FoVs, sensing radius affects the percentage of the area coverage is shown.

In [7], the Weifang Cheng et.al. Proposed distributed greedy algorithm MDAC (maximum directional area coverage) also termed as DGreedy to maximize the area by scheduling working direction of the sensor. MDAC is proved as NPComplete problem. In DGreedy algorithm the numbers of the neighboring nodes( nodes having overlapping area are neighbor nodes) are chosen as priority. In case of ties the geographical co-ordinates are used. For irregular region the grid based application similar to [8].The higher priority nodes decides their working direction in the direction that is not covered by the higher priority nodes.

In [9], the MDAC problem is analyzed and two greedy algorithm have been proposed .The proposed two algorithm are update priority and least overlapped area first. Update priority uses different scheduling algorithm from DGreedy In update priority the priority depends on the unscheduled neighbor and updated dynamically whereas in DGreedy algorithm priority depends on the least number of neighbors' and after deciding the working direction rest all sensors follow the estimated scheduling order. After scheduling, the working directions are scheduled which depends on the two factors:

- Least covered region

- Least overlapped region.

In LOF (least overlapped area first), the priority depends on the least overlapped area i.e. the area that has minimum overlapping FoVs. The node after communicating coverage message decides the priority. After every scheduling, nodes maintain the values of the covered region and overlapped area.

In [10], the algorithm update priority and least overlapped area first defined in [9] are improved by using rotatable sensors. The LOFR (least overlapped area first with rotatable) uses least overlapped region as priority measures .Unlike LOF, LOFR rotates the nodes working direction to least covered area or least overlapped region whereas in UPR (update priority with rotatable) the nodes decide the rotating angle in every round and follow the same procedure as update priority of [9]. The nodes rotates and change their working direction if there is any overlapping.UPR outperforms in terms of sensing radius.

In [11], a face away algorithm is defined that schedules each and every node to the center line of sight of their maximum angle bisector of the adjacent nodes.
After studying and analyzing the previously done works; the results and their respective coverage rates ( for 200 sensor nodes) can be categorized as:

Table 1. Results drawn from previous studies

\begin{tabular}{|l|l|}
\hline Algorithm & Coverage rate \\
\hline Random & $67.6 \%$ \\
\hline Face away & $72.04 \%$ \\
\hline MDAC & $74.19 \%$ \\
\hline $\begin{array}{c}\text { Update priority } \\
\text { Least overlapped area } \\
\text { first }\end{array}$ & $76.34 \%$ \\
\hline $\begin{array}{c}\text { Update priority with } \\
\text { rotatable }\end{array}$ & $84.16 \%$ \\
\hline $\begin{array}{c}\text { Least overlapped area } \\
\text { first with rotatable }\end{array}$ & $79.68 \%$ \\
\hline
\end{tabular}

The conclusion that can be drawn from the above table is as follows (from the survey that have been done):

1) Update priority provide $2.15 \%$ improvement over MDAC.There is little improvement because of ties due to the same priority.

2) Least overlapped area first provides $6.44 \%$ improvement because as least the overlapped area therefore the more will be the coverage.

3) Update priority with rotatable $12.12 \%$ improvement.

4) Least overlapped first with rotatable outperforms $7.64 \%$ better than face away.

It is proved that Coverage rate is directly proportion to the network size i.e. greater the network size lesser will be the coverage rate.

On the basis of the coverage rate the above algorithms can be arranged in ascending order as follows:

Random<Face away< MDAC < Update priority<Least overlapped first with rotatable $<$ Least overlapped area first<update priority with rotatable.

\subsection{Maximum Coverage with Minimum Sensors}

The study of network is done as the possibility to cover the each target by at least one sensor node and to cover the entire network.

Yahya Osais et. al. [12], defines minimum sensor placement problem and also formulate the an ILP which is generalization of grid coverage problem and isotropic MSP (minimum sensor placement) problem defined in [13, 14, 15].The ILP model is used to minimize the need of sensor network required for monitoring the sensor network. The results shows that the greater will be the placement sites the greater will be the percentage reduction in number of sensors. 
For the purpose of elaborative study, Jing Ai et.al. [3], propose a Maximum Coverage with Minimum Sensors (MCMS) problem in which coverage in terms of the number of targets to be covered is maximized whereas the number of sensors to be activated is minimized. A TIS (Test in Sector) test is designed to define a relationship between sensor, orientation and a target. It defines Maximum coverage with sensor problem that contain $\mathrm{s}$ a set of targets and $n$ homogenous set of sensors with p possible orientation and derive ILP to give optimal solution for MCMS instance for small instance, CGA(Centralized Greedy Algorithm) and DGA (Distributed Greedy Algorithm).In CGA nodes have communication overhead and make locally optimal decision about its state and orientation whereas in DGA nodes make global decision and it terminates in Finite time as well as generate no hidden targets assuming static priorities.

The author also presents SNCS (sensing neighborhood cooperative sleeping protocol) an extension to DGA to provide dynamic scheduling among sensor nodes depending on the amount of residual energy. SNCS performs adaptive scheduling. Nodes set themselves as active or inactive in order to save energy and for prolong network lifetime.

\section{CONCLUSION}

It can be observed that by increasing the number of directional sensor nodes the coverage area can be enhanced but it is not the desirable case. Therefore, a trade-off between the number of nodes deployed and the coverage rate must be considered.

From the above discussion it can be concluded as follows:

- Increasing the sensing radius, increases the number of the active sensors and the coverage rate.

- Increasing the number of possible orientations, decreases the number of active sensors and coverage rate while increases the number of sensor nodes.

- Greater FoV results in lesser number of nodes and less number of orientations.

\section{REFERENCES}

[1] C.F. Huang and Y.C. Tseng, "The coverage problem in a wireless sensor network," In ACM International Workshop on Wireless Sensor Networks and Applications (WSNA), 2003, pp. 115-121.

[2] Xiaofeng Han, Xiang Cao, Errol L. Lioyd, Chien-Chung Shen,"Deploying Directional Sensor Network with Guaranteed Connectivity and Coverage", Preliminary work of this paper has appeared as a two-page poster in IEEE INFOCOM 2007.

[3] Habib Mostafaei, Mohammad Reza Meybodi,"An Energy Efficient Barrier Coverage Algorithm for Wireless Sensor Networks", published in Wireless Pers Commun DOI 10.1007/s11277-014-1626-1, on 31 January, 2014.

[4] Dimple Bajaj, Manju, "Maximum Coverage Heuristics $(\mathrm{MCH})$ for Target Coverage Problem in Wireless Sensor
Network”,published in 978-1-4799-2572-8/14/\$31.00_c 2014 IEEE.

[5] Giordano Fusco and Himanshu Gupta," Selection and Orientation of Directional Sensors for Coverage Maximization", published in IEEE.

[6] Jian Wang, Changyong Niu, Ruimin Shen," Prioritybased target coverage in directional sensor networks using a genetic algorithm", published in 0898-1221/\$ see front matter 2008 Elsevier Ltd. All rights reserved.doi:10.1016/j.camwa.2008.10.019.

[7] Weifang Cheng Shanshan Li Xiangke Liao Shen Changxiang Haitao Chen, Maximal Coverage Scheduling in Randomly Deployed Directional Sensor Networks",published in 2007 International Conference on Parallel Processing Workshops (ICPPW 2007), 07695- 2934-8/07 @ 2007,IEEE.

[8] H. Chen, H. Wu, N. Tzeng, "Grid-based Approach for Working Node Selection in Wireless Sensor Networks,"Proceedings of IEEE International Conference on Communications",Paris, France, 2004

[9] Chiu-Kuo Liang, Chih-Hung Tsai, Meng-Chia He,’On Area Coverage Problem in Directional Sensor Network", published in 978-1-61284-663-7/11/\$26.00 (C)2011 IEEE.

[10] Chiu-Kuo Liang, Chih-Hung Tsai, Ting-Hao Chu," Coverage Enhancing Algorithms in Directional Sensor Networks with Rotatable Sensors", published in 978-07695-4624-7/11@ $2011 \quad$ IEEE DOI 10.1109/APSCC.2011.41.

[11] Kandoth, C., Chellappan, S., "Angular Mobility Assisted Coverage inDirectional Sensor Networks," to be appeared in International Conference on NetworkBased Information Systems, NBIS 2009.

[12] Yahya Osais, Marc St-Hilaire, and F. Richard Yu," On Sensor Placement for Directional Wireless Sensor Networks",published in 978-1-4244-3435-0/09/\$25.00 (C)2009 IEEE.

[13] K. Chakrabarty, S. Sitharama, H. Qi, and E. Cho, "Grid coverage for surveillance and target location in distributed sensor networks," IEEE Transactions on Computers, vol. 51, no. 12, pp. 1448-1453, 2002.

[14] S. Sahni and X. Xu, "Algorithms for wireless sensor networks," International Journal of Distributed Sensor Networks, vol. 1, no. 1, pp. 35-56, January 2005.

[15] X. Xu and S. Sahni, "Approximation algorithms for sensor deployment," IEEE Transactions on Computers, vol. 56, no. 12, pp. 1681-1695,December 2007.

[16] Jing Ai · Alhussein A. Abouzeid," Coverage by directional sensors in randomly deployed wireless sensor networks", appeared in Springer Science+Business Media, Inc. 2006 on 25 october 2005. 